\title{
34. COMMISSION DE LA MATIERE INTERSTELLAIRE ET \\ DES NEBULEUSES PLANETAIRES
}

\author{
Report of Meeting, 26 and 29 August 1967
}

President: S. B. Pikelner.

SeCRETARY: T.K. Menon.

\section{Business Meeting}

At the first meeting on 26 August the Draft Report was approved subject to minor corrections or additions.

The President announced that the present Vice-President D.E. Osterbrock will be the next President and the next Vice-President will be F.D. Kahn. The following were suggested as the members of the Organizing Committee: L. H. Aller, J. M. Greenberg, L. Rosino and S. B. Pikelner. S.A. Colgate was suggested as a Consulting Member.

\section{Scientific Meetings}

\section{INTERGALACTIC GAS}

$R$. A. Syunyaev reviewed the properties of intergalactic gas from the theoretical and the observational points of view. He suggested that the intergalactic gas is most probably in the form of ionized hydrogen and helium. He pointed out that the observations so far have only suggested an upper limit of $P_{\text {gas }}<4 \times 10^{-29} \mathrm{gms} / \mathrm{cm}^{3}$ which is still too high compared to the critical matter density in the universe of about $2 \times 10^{-29} \mathrm{gm} / \mathrm{cm}^{3}$.

Y.B. Zel'dovic reported on attempts to measure intergalactic radiation in the bands $\lambda 1050$ $1340 \AA$ and $\lambda 1225-1340 \AA$ using two detectors on the Interplanetary Probe Venus II. The first band includes unshifted Ly $\alpha$ and the second band observes gas at a great distance such that $\lambda 1216$ $(1+Z)$ should go into that region. Theory predicts intensity as a function of $P_{\text {gas }}$ and temperature. The upper limit from observations $\mathrm{I}<10^{-7} \mathrm{ergs} / \mathrm{cm}^{2} / \mathrm{sec} /$ Steradian (Kurt and Synyaev).

G.B. Field stressed that the information on neutral intergalactic gas depends on the cosmological hypothesis. His calculations on the abundance of $\mathrm{H}_{2}$ molecules indicated that it is low but not negligible. He emphasized the need to repeat Kohler's observations of the $21-\mathrm{cm}$ absorption in the Virgo Cluster.

The Nancay group reported on their repetition of Kohler's observations with, so far, negative results.

$J$. Baldwin discussed the attempts at Cambridge to detect the step in the spectrum at about $1420 \mathrm{Mc} / \mathrm{s}$ expected from the $21-\mathrm{cm}$ radiation of intergalactic neutral atomic hydrogen. The upper limit for $\mathrm{H} I$ is $\leqslant 8 \times 10^{-30} \mathrm{gm} / \mathrm{cm}^{3}$.

Field pointed out that at the low densities involved the spin temperature of atomic hydrogen is in equilibrium with the cosmic background and hence there is no reason to expect significant radiation from them.

C. Varsavsky reported on unsuccessful attempts by W. G. Popped to detect $21-\mathrm{cm}$ radiation from Hoffmeister's extragalactic absorbing cloud believed to be at a distance of $10^{5}$ to $10^{6}$ parsecs.

Syunyaev remarked that, since the U.V. spectrum of quasars is relatively flat, the absorption by $\mathrm{H} I$ is more critical and hence the upper limit can be better determined in the case of such observations. 


\section{MAGNETIC FIELD IN THE GALAXY}

R.D. Davies presented a review of the present status in our knowledge of the magnetic field in the spiral arms.

Recent measurements of the polarized background radio emission of the Galaxy and of the Faraday rotation of the linearly polarized radiation from extragalactic radio sources on passing through the Galaxy, have made a significant contribution to our understanding of the magnetic field in the Galaxy. These data can be combined with information on the polarization of starlight, on the synchrotron emission from the galactic disk and on the Zeeman effect in interstellar clouds to provide a new picture of the magnetic field structure in the local spiral arm.

The field in the local arm appears to be directed towards $l^{1 \mathrm{II}}=70^{\circ}$ and there is evidence that the field is converging in this direction. In addition the field at intermediate latitudes above and below the plane is directed in opposite senses. The field below the plane is directed towards $l^{11}=70^{\circ}$ approximately. At present it is not clear whether this field is associated with the spiral arm as a whole or is a more local phenomenon. Irregular magnetic field structures are found in the spiral arm. The largest are the 'spurs' of synchrotron emission which contain fields aligned parallel to the outer edge of the 'spurs'. In addition a fine structure seems to be intrinsic to the field. Studies of the optical polarization of starlight in galactic clusters and of the depolarization of radio emission from the extragalactic sources at low latitudes indicate a structure of about $0.3 \mathrm{pc}$.

An estimate of the mean strength of the magnetic field along the spiral arm can be obtained from the Faraday rotation data of extragalactic sources. The value which is about $5 \times 10^{-6}$ gauss is dependent on the assumed clumpiness of the electron density in the spiral arm. This is much lower than $3 \times 10^{-5}$ gauss normally estimated for the field strength necessary to produce the observed synchrotron emission from the galactic disk. The difference between these values again is consistent with a tangled field on the small scale which nevertheless has a net mean value.

Seymour discussed his spherical harmonic analysis of the data on Faraday notation, optical polarization and background polarization. His results are reported in M.N.

H.C.Van de Hulst pointed out that one can make estimates of magnetic field based on cosmic ray data alone.

S.B. Pikelner remarked that a tangled magnetic field tends to increase the velocity dispersion which is not observed. Hence the reversals of magnetic field will have to be on a large scale.

$J$. R. Shakeshaft suggested that, if the curvature of the radio-frequency spectrum of the galactic background is related to the steepening of the cosmic ray electron energy spectrum beyond $3 \mathrm{GeV}$, then we get a value of the magnetic field to be $5 \times 10^{-6} \mathrm{G}$.

\section{DYNAMICS OF GAS CLOUDS}

G. B. Field reviewed the results of the Field-Saslaw model of interstellar cloud mass spectrum. For $\sigma \varepsilon$ independent of mass, the mass spectrum is predicted to be a power law $m^{-\beta}$, with $\beta=3 / 2$. Recent work by Field and Hutchins shows that if $\sigma \varepsilon$ depends on mass as expected, if all clouds have the same density and if there is equi-partition of energy, $\beta \simeq 3 / 2$, contrary to initial expectation.

These predictions are compared with observations. $21-\mathrm{cm}$ observations by Heiles disclose some 800 'cloudlets' $\left(1<m<30 \mathrm{M}_{\odot}\right)$ and 12 'concentrations' $(300<m<\infty)$. There are no objects in the range $30-300 \mathrm{M}_{\odot}$ contrary to prediction. Moreover, the cloudlets follow a power law closely, with $\beta=1.65$. The relative numbers of cloudlets and concentrations are characterized by $\beta=1 \cdot 25$.

Observations of dust and $\mathrm{Ca}^{+}$absorption also show that a range of masses is present. According to Scheffler statistics of color excesses of 4700 stars indicate $\beta=2 \pm 0 \cdot 2$. Therefore there is some degree of agreement between the model $(\beta=3 / 2)$ and the observations $(\beta=1 \cdot 65-2)$. Nevertheless, there are considerable problems. On the observational side, there is the question of the clouds in the mass range $30-300 \mathrm{M}_{\odot}$. On the theoretical side, there is the question of fragmentation of clouds during collision (ignored in the model) and residual elasticity of cloud collisions due to magnetic 
field (also ignored in the model). Various mechanisms for cloud formation, including thermal and hydromagnetic instability were discussed.

$P . V$. Sčeglov reported that his studies using a narrow band Fabry-Perot etalon, with a bandwidth of less than $3 \mathrm{Km} / \mathrm{sec}$ at $\mathrm{H} \alpha$, indicated existence of high velocities in the range of -120 to $+150 \mathrm{Km} / \mathrm{sec}$ in a number of emission nebulae.

M. Smith reported on his measurements of the line profiles of $\mathrm{H} \alpha$ and [N II] lines in a number of emission nebulae using a Fabry-Perot technique.

H. Zimmermann discussed the processes occurring during the collision and cooling of interstellar clouds. He considered in turn the initial compression period, then the subsequent expansion and the interval between collisions. His calculations suggest a mean number density of $\mathrm{H}_{2}$ molecules of at most $5 \times 10^{-4}$ times the number density of hydrogen atoms. The details of his theory are published in Astronomische Nachrichten.

S.B. Pikelner stressed the importance of magnetic fields in such considerations. Motion of the clouds along the magnetic field is possible but perpendicular to the field lines only small amplitude oscillations are possible. He suggested that the collisions perpendicular to the galactic plane are elastic but along the lines collisions can make clouds grow. Heavy clouds bend magnetic lines down and collect clouds, until new stars are formed.

\section{INTERSTELLAR GRAINS}

T.P. Stecher reviewed observations pertaining to interstellar grains.

'The wavelength dependence of interstellar extinction has been extended from the far infrared to the rocket ultraviolet with the result that no proposed particle will satisfactorily reproduce the observations with the existing theoretical treatments. Regional extinction curves have been extensively studied and show a large variation in the $\mathrm{R}$ value. There is some suggestion, however, that this may be a property of very young objects and for the general interstellar medium $\mathbf{R}$ may be more nearly a constant.

'Excellent observations of both color and polarization angle have been made on several reflection nebula which should reveal the angular scattering dependence of the particles when given a thorough theoretical treatment. In the special case of backscattering the theoretical interpretation is greatly simplified. The diffuse galactic light has been measured by several observers and the results seem to indicate particles that are isotropic scatterers with a high albedo. This appears contrary to the theoretical properties of previously proposed particles.

'The wavelength dependence of interstellar polarization shows a considerable scatter from star to star but the maximum polarization usually occurs in the yellow. A wavelength dependence on the direction of the magnetic field appears to be established. The dependence of position angle on color is not clear at this time. The correlation of polarization with color excess shows scatter and indicates there is a maximum degree of polarization.

'The infrared ice band at $3.05 \mu$ was not observed although it should have been if ice is a major component of the interstellar grains. The Mira variables have been found to be intrinsically polarized lending support to the hypothesis that grains are formed in late type stars. Some infrared observations have been interpreted as thermal emission of grains heated by young stars embedded in the clouds. The temperature of these grains is over $10^{3} \mathrm{degK}$.

'The diffuse interstellar lines now number 28 and remain unidentified. Accurate profiles of $\lambda 4430$ have been obtained. This line was found not to correlate physically with the change of slope at 4300 in the extinction curve. $\lambda 4430$ does, however, show a correlation with extinction with a different line for high velocity clouds.

'The work of the last three years tends to reject ice and support some form of carbonaceous material. The observation pertaining to the interstellar grains as interpreted by existing theoretical models are, however, inconsistent and we must therefore conclude that the composition of the grains is unknown. Whether the difficulty lies in the theory and its approximations and measured physical parameters or the observations or both remains to be seen. We can, however, be hopeful 
that the next three years could provide the solution or at a minimum considerable additional progress.'

J. Mayo Greenberg reviewed theories of interstellar grains.

'During the past few years the observation of extinction, polarization and scattering by interstellar grains has been very considerably extended and this faces us with the requirement of more definitive theories.

'The theoretical problems may be put into two categories: (1) direct confrontation with observation. (2) physical and chemical interactions of grain with stars and with the interstellar medium. Although the latter are often only indirectly related to observations they are the ultimate foundation on which all theories must be based. An apparently satisfactory grain model-from an optical point of view-may be entirely inconsistent with physical or chemical criteria. (As an absurd example consider the motion that the grains are made of uranium hexofluoride particles.) Problems of direct confrontation include interstellar extinction and polarization, light from reflection nebulae, diffuse galactic light, grain emission in the infrared. Physical and chemical problems include theories of grain growth, interactions with stellar radiation, interactions with the interstellar gas atons and ions, interaction with low energy cosmic rays (superthermal particles), interactions with magnetic fields. Finally there are problems which are sufficiently uncertain that they are difficult to categorize. In particular, attention is directed to the diffuse interstellar lines which are almost certainly intimately connected with the grains.

'No attempt will be made here to discuss all of the recent theoretical work. The Draft Report of Commission 34 has a rather complete survey of the literature in this field. We limit ourselves here to only a few points.

'Very extensive calculations have been carried out on model interstellar grains consisting of graphite cores with dielectric mantles or purely dielectric elongated (infinite cylinder) grains. Detailed considerations have been given to the optical properties of graphite and dirty ice throughout the spectral range of astronomical observations. It now seems that both the graphite core plus dielectric mantle and the elongated dielectric grains can give a reasonable representation of the interstellar extinction from the far infrared to about $\lambda^{-1}=5 \mu^{-1}$. Beyond this region (toward the far U.V.) neither model seems to predict a continued rise in the extinction as has been reported by Stecher. The reason for this lies in the fact that the dielectric materials which one can conceive of as either existing by themselves or as growing on graphite cores have a high absorptivity in the far U.V. In this wavelength region the grains are relatively large compared with the wavelength and consequently give rise to a reduced extinction relative to similar sized particles which are nonabsorbing. In the far infrared (below $\lambda^{-1}=0.5 \mu^{-1}$ ) the very anomalous types of extinction which have been suggested can not be produced by normal sized grains nor even by moderate modifications in the sizes (or size distributions) of ordinary grains as might be produced by interaction of the grains with stellar fluxes in $\mathrm{H}_{11}$ regions.

'The observed maximum polarization relative to extinction can be matched by graphite coredielectric mantle particles as well as by dielectric cylinders. The latter type of particles have been shown to give a moderately good representation of the wavelength dependence of polarization but with two difficulties which are not yet completely resolved; namely, the predicted position of the maximum polarization is a bit off and the sharp polarization drop in the infrared is not reproduced. Explanations of these discrepancies may possibly be found in the variations of degree of orientation with size. The flat graphite by itself can not produce the wavelength dependence of polarization. This has been shown by calculations for the small flakes and for larger flakes by use of microwave scattering results (unpublished). For graphite core ice mantle particles approximate calculations indicate that the polarization dependence on wavelength may be reasonably given. However microwave experiments on such particles indicate that the theory may not yet be reliable and this result is therefore questionable.

'A very significant advance in our understanding of the Davis-Greenstein magnetic orientation mechanism has been made by Jones and Spitzer. On the basis of application of this theory to the orientation of dirty ice grains and to the orientation of graphite core-ice mantle grains it may now 
be stated that: (1) For dielectric grains whose magnetic susceptibility is as estimated by Davis and Greenstein the magnetic field required to produce the highest observed polarization is $B=2 \times 10^{-5}$ gauss; (2) for graphite core-ice mantle grains the minimum magnetic field requirement is higher by perhaps a factor of three. Using "super paramagnetism" the magnetic field requirement for dielectric grains may be reduced by orders of magnitude.

'Among the unsolved problems the explanation of the diffuse interstellar lines is outstanding and we can hope that when we know their origin we will have better defined the interstellar grains.

'Further observations of interstellar extinction and polarization in the ultraviolet beyond $\lambda^{-1}=3 \mu^{-1}$ are clearly indicated. Metallic and graphite particles will produce significantly less polarization in this region than dielectric ones. Beyond $\lambda^{-1}=5 \mu^{-1}$ the situation does not appear to be so distinctively different. Observations and theoretical calculations of the scattering and extinction by grains in filamentary structures of reflection nebulae would be extremely desirable because here one needs to explain simultaneously the principal optical properties of the interstellar grains. The answer to the question of orientation of grains by possible magnetic fields defining these filaments is of considerable interest.

'Finally and without discussion we list some additional problems for future work where present solutions are either non-existent or inadequate: interaction of superthermal particles with the grains, effects of surface or general irregularities on the optical characteristics of small particles, the chemistry and physics of the origin and growth of interstellar particles, detailed studies of grains in $\mathrm{H}$ II regions.'

\section{Appendix \\ ADDENDA AND CORRIGENDA TO THE REPORT OF COMMISSION 34 PUBLISHED IN TRANSACTIONS IAU, VOLUME XIII A}

On page 730 , paragraph 2 , line 5, after: '...on the central star (4)', add: 'Kohoutek (4a) identified 109 new faint planetaries, and positions of 322 planetaries were measured ( $4 \mathbf{b})$ '.

On page 731, at the end of paragraph 5 beginning with: 'A central star of WN type...', add 'Variability of nuclei of nebulae was studied by Kohoutek $(66 \mathbf{a}, \mathbf{b})$ '.

On page 740, at the end of paragraph 1 after: '... in Cygnus Loop.', add: 'Poveda (241a) argued that filaments are thin sheets. The total energy in the beginning evaluated as high as $5 \cdot 10^{49}$ ergs.'

On page 744 , at the end of paragraph 2 , after: '... and no nucleus forms.', add: 'Collapse of isothermal gas sphere was studied and used to formations of stars clusters, quasars and galaxies (327a).'

On page 745, in the Bibliography, the following references should be added:

4a. Kohoutek, L. $\quad$ 1965, Bull. astr. Inst. Csl., 16, 221.

4b. Kohoutek, L., Pěkný, Z., Perek, L. 1965, Bull. astr. Inst. Csl., 16, 189.

13a. Kohoutek, L. $\quad$ 1964, Publ. astr. Soc. Pacif., 76, 435.

66a. Kohoutek, L. $\quad 1964$, Bull. astr. Inst. Csl., 15, 161.

66b. Kohoutek, L. 1966, Bull. astr. Inst. Csl., 17, 318.

106a. Griem, H. R. $\quad 1967$, Astrophys. J., 148, 547.

241a. Poveda, A. $\quad$ 1964, Ann. Astrophys., 27, 522.

327a. Penston, M. V. $\quad 1966$, R. Obs. Bull., no. 117, E 300. 